\title{
Overweight and Normal-Weight Children's Decision-Making in a Child Variant of the Iowa Gambling Task
}

\author{
Nele Lensing and Birgit Elsner \\ Developmental Psychology, University of Potsdam, Karl-Liebknecht Str. 24-25, 14476 Potsdam, Germany \\ Correspondence should be addressed to Nele Lensing; lensing@uni-potsdam.de
}

Received 15 June 2017; Revised 16 September 2017; Accepted 26 October 2017; Published 16 November 2017

Academic Editor: Glenda Andrews

Copyright (c) 2017 Nele Lensing and Birgit Elsner. This is an open access article distributed under the Creative Commons Attribution License, which permits unrestricted use, distribution, and reproduction in any medium, provided the original work is properly cited.

\begin{abstract}
In the Iowa Gambling Task (IGT), overweight as compared to normal-weight adults make more risky decisions resulting in immediate rewards and long-term losses. Findings regarding a potentially moderating role of gender have been inconsistent and investigations on the development of weight-group differences in decision-making during childhood are lacking. Using a 3-wave longitudinal study, we examined decision-making in a matched sample of 94 overweight and 94 normal-weight children (49\% girls, aged 6-9 years at wave 1) over a 3-year period. Decision-making was measured with the Hungry Donkey Task (HDT), an ageappropriate version of the IGT, and learning within the task was examined via analysis across trial blocks. Mixed-design ANOVA revealed that more risky decisions were made by overweight as compared to normal-weight children and by girls as compared to boys. Within-task learning was evident at all three waves, moderated by weight group and gender. However, although risky decisions generally decreased across the 3-year period, weight-group and gender differences did not significantly change over the 3 -year period. Our findings demonstrate that weight-group and gender differences in decision-making are already present and do not significantly change over a 3 -year period in middle childhood.
\end{abstract}

\section{Introduction}

The prevalence of overweight and obesity has severely increased over the last three decades in both children and adults [1]. In the so-called obesogenic environment, where palatable, high-calorie food is almost always available, energy intake is based to a great extent on individual food choices [2], and it requires self-regulated decision-making to maintain a healthy energy balance. Therefore, the role of decisionmaking in the development of overweight has gained research interest during the last decade.

Food choices and other daily-life decisions are often characterized by competing goals, for example, more shortterm hedonic goals like enjoying a palatable snack and rather long-term goals like keeping a healthy body weight [3]. Furthermore, some consequences of a particular behavior, such as eating a slice of cake, are uncertain or distant (e.g., weight gain does not follow immediately), whereas other consequences yield immediate gratification (e.g., a pleasant feeling), making it difficult to choose what is advantageous in the long term. In a developmental perspective, young children's decisions are based primarily on short-term outcomes [4], and this tendency decreases throughout childhood until late adolescence [5]. A key structure involved in decision-making under uncertainty (i.e., with unknown outcomes) is the orbitofrontal cortex (OFC) [6], a region that shows specific structural and functional variations in overweight/obese as compared to normal-weight children and adults [e.g., $[7,8]]$. Moreover, a reduced OFC volume has been associated with (un)healthy food choices [9], so a potential association between decision-making deficits and overweight might be mediated by this brain region.

A widely used measure for decision-making under uncertainty is the Iowa Gambling task (IGT) [10], in which participants try to gain a maximum number of points by drawing cards from four decks. Unknown to the participant, two of the decks yield not only relatively large gains but also relatively high unpredictable losses, resulting in a long-term net loss ("disadvantageous choices"), whereas the other two decks yield smaller gains but also smaller losses, resulting in a longterm net gain ("advantageous choices"). Usually a net score is calculated, subtracting the number of disadvantageous 
choices from the number of advantageous choices [10]. Lower net scores indicate a stronger preference for high immediate reward and insensitivity to negative long-term consequences. IGT net scores can be analyzed based on all trials (overall net score) or blockwise, dividing the task in several blocks of trials. A blockwise analysis allows examining learning within the task, which is based on the feedback about gains/losses obtained from previous card choices [11].

Studies that used the IGT found consistent evidence for impaired decision-making under uncertainty, evident in relatively more disadvantageous choices, in overweight and obese as compared to normal-weight adults [e.g., $[12,13]]$. Likewise, obese and overweight children and adolescents show inferior executive functioning than do their normal-weight peers [7]. However, only few studies with children and adolescents have investigated weight-group differences in decision-making under uncertainty, as measured with the IGT, so it is unclear at which age the weight-group differences develop. The few studies that have used the standard version of the IGT [11] or a child-appropriate analogue, like the Hungry Donkey Task (HDT, see Method) [5, 14], found weight-group differences in within-task learning in 11- to 16-year-olds, evident in a relative decrease of disadvantageous choices across trial blocks. Overweight (pre)adolescents learned to a lesser extent than their normal-weight peers to use the feedback from previous trials to shift to the more advantageous choices.

In a large sample of 6- to 11-year-olds, however, weightgroup differences in HDT net scores were found just in the first of two waves, which were approximately one year apart [15]. In that study, only the overall net score (minus the first 10 trials) was analyzed, neglecting the within-task learning progress that can differ between individuals and groups. Noteworthy, the overweight group had a much smaller sample size and also lower SES as measured via maternal education than the normal-weight group, which might have affected the results. The present study therefore aimed at investigating weight-group differences in the withintask learning of decision-making under uncertainty across 3 years during middle childhood in a sample of overweight and normal-weight children matched for age, gender, and SES.

SES and parental education level are associated with both weight status [16] and performance in the IGT $[17,18]$. However, only few studies have controlled for these possibly confounding variables, and conclusions regarding their role were inconsistent $[18,19]$. Gender differences in IGT performance have been found consistently in adults, with males outperforming females most of the times [20]. Although these findings might suggest otherwise, only few studies on weight-group differences in the IGT have taken gender into account. In fact, most of the studies included only female participants or analyzed women and men together [12]. Two studies that compared men and women yielded inconsistent results, indicating that, on one hand, obese women but not obese men show a larger preference than normal-weight controls for high immediate reward and insensitivity to rare but high losses [8]. On the other hand, only in men but not in women, overweight and obesity were associated with a preference for high immediate rewards and insensitivity to frequent losses [22]. The marked differences in the direction of effects may be explained by characteristics of the used modified versions of the IGT, which focused on various aspects of decision-making, that is, (in)sensitivity to rare losses [8] versus (in)sensitivity to frequent losses [22]. Gender has also been found to moderate weight-group differences in decision-making measured by delay discounting, with obese women showing a larger preference for immediate reward than normal-weight women, but there were no such differences in obese versus normal-weight men [23]. To our knowledge, so far no studies have systematically investigated gender differences in overweight and normal-weight children's decision-making, yet the reported findings in adults emphasize the need to include gender as a possibly moderating variable.

During elementary school, the prevalence of overweight increases, indicating that middle childhood is a critical period for the development of overweight and obesity [21]. Entry to elementary school entails many changes in children's life style, including more autonomous food choices: when eating at school, children are less supervised as compared to eating at home or at kindergarten. In addition, many elementary school-aged children receive a regular pocket money [24], which they can use to purchase snacks and beverages. Such changes increase the demand for self-regulation and foodrelated decision-making. Even though this age period is likewise associated with growth in self-regulation abilities [25, 26], individual differences in these abilities can either support or challenge a healthy food intake.

The present study investigated weight-status- and genderrelated differences in within-task learning of decisionmaking in a child-appropriate version of the IGT, using a longitudinal design (three waves, T1, T2, and T3, over a 3-year period) in middle childhood. In a sample of 94 overweight and 94 normal-weight children (6 to 9 years old at T1) matched for age, gender, and SES, we used a 60-trial HDT and examined learning in three blocks of 20 trials each. All children were in one of the first years of elementary school (grade 1 to grade 3 ) at $\mathrm{T} 1$ and in one of the last years of elementary school (grade 4 to grade 6) at T3 (in the federal state of Brandenburg (Germany), elementary school includes grades 1 to 6 (age range: 5 to 13 years)). We hypothesized, first, that overweight children would be less able than normalweight children to refrain from high immediate rewards and would therefore continue to make relatively more disadvantageous choices (resulting in a lower net score) till the end of the task. Second, we expected the weight-group differences to occur not in the first block but only in later blocks, indicating better use of feedback from previous trials in normal-weight as compared to overweight children [11, 14]. Third, we assumed that the weight-group differences in decision-making would become larger over the 3-year period, given the eating-related lifestyle changes in middle childhood and the increased demand for self-regulation. Fourth, we explored a possibly moderating role of gender in the weightgroup differences, but given the inconsistent findings in adults $[8,22]$, we had no specific hypothesis regarding the gender effect. To rule out possible confounding effects of age and SES, we also matched the two weight groups with regard to these two variables. Moreover, we tested whether 
TABLE 1: Means (and SDs) of control variables for weight groups and for boys and girls.

\begin{tabular}{|c|c|c|c|c|c|c|c|c|}
\hline & $\begin{array}{c}\mathrm{NW} \\
(n=94)\end{array}$ & $\begin{array}{c}\text { OW } \\
(n=94)\end{array}$ & $t$ & $p$ & $\begin{array}{c}\text { Girls } \\
(n=90) \\
\end{array}$ & $\begin{array}{c}\text { Boys } \\
(n=98) \\
\end{array}$ & $t$ & $p$ \\
\hline Age (T1) & $8.35(0.78)$ & $8.36(0.79)$ & 0.12 & .90 & $8.26(0.72)$ & $8.44(0.82)$ & 1.52 & .13 \\
\hline Age (T2) & $9.09(0.78)$ & $9.12(0.80)$ & 0.21 & .84 & $9.02(0.74)$ & $9.18(0.83)$ & 1.33 & .19 \\
\hline Age (T3) & $11.07(0.80)$ & $11.11(0.81)$ & 0.36 & .72 & $11.02(0.77)$ & $11.16(0.84)$ & 1.33 & .23 \\
\hline SES (T1) & $2.68(0.96)$ & $2.66(0.97)$ & 0.15 & .88 & $2.63(0.92)$ & $2.7(1.00)$ & 0.50 & .62 \\
\hline BMI-SDS (T1) & $0.01(0.57)$ & $1.93(0.39)$ & 26.88 & $<.001$ & $0.99(1.10)$ & $0.95(1.06)$ & 0.25 & .80 \\
\hline BMI-SDS (T2) & $0.08(0.59)$ & $1.94(0.36)$ & 26.29 & $<.001$ & $1.00(1.09)$ & $1.02(1.03)$ & 0.11 & .92 \\
\hline BMI-SDS (T3) & $0.08(0.62)$ & $1.95(0.38)$ & 24.82 & $<.001$ & $1.00(1.11)$ & $1.02(1.04)$ & 0.18 & .86 \\
\hline $\mathrm{PS}(\mathrm{T} 1)^{1}$ & $52.13(8.03)$ & $50.22(9.37)$ & 1.49 & .14 & $52.06(8.45)$ & $50.36(9.00)$ & 1.32 & .19 \\
\hline
\end{tabular}

Note. Results of two-tailed $t$-tests. OW: overweight group; NW: normal-weight group; T1, T2, and T3: data waves; SES: socioeconomic status; BMI-SDS: standardized BMI score; PS: processing speed. ${ }^{1} n_{\text {total }}=185$.

the weight groups or gender groups differed in processing speed and additionally controlled for this variable in the analyses.

\section{Method}

2.1. Participants. The data was taken from the "PIER study," a larger longitudinal study on intrapersonal developmental risk factors in childhood and adolescence in the federal state of Brandenburg (Germany). In the overweight group, we included all children from the original sample of 1,660 children who were overweight (weight-group classification (see description of Body Weight Status)) at all three waves. For each overweight child, a "statistical twin" who had normal weight at all three waves was drawn from the original sample, based on same gender, same SES score (see description of Socioeconomic Status), and same age $( \pm 0.05$ years). This resulted in a matched sample of 94 overweight and 94 normal-weight children (in both groups: 45 girls and 49 boys) aged from 6.88 to 9.88 years at $\mathrm{T} 1$ ( $\mathrm{M}=8.35$ years; $\mathrm{SD}=0.78)$.

As can be seen in Table 1, the matching procedure was successful in that age, SES score, and processing speed did not differ significantly between the two weight groups or between girls and boys. Yet, as intended, body weight measures (BMISDS, see description of Body Weight Status) [27] differed significantly between the overweight and the normal-weight groups but not between girls and boys.

\subsection{Measures}

2.2.1. Body Weight Status. Children's body weight and height (without shoes, hats, and jackets) were assessed using calibrated digital scales and ultrasound measurement devices, respectively. In order to address age- and sex-specific alterations in body fat mass, we used BMI percentiles based on German reference data [27] as cut-off points for the weightgroup classification. To the overweight group, we assigned all children who had a BMI above the age- and sex-specific 90th percentile at all three waves [28]; the normal-weight group consisted of children with a BMI between the 10th and 90th BMI percentiles at all three waves. In the overweight group, the numbers of children classified as overweight (BMI between 90th and 97th percentiles) were at T1 $n=46(n$ girls/boys $=20 / 26)$, at T2 $n=47(20 / 27)$, and at T3 $n=42$ $(18 / 24)$, and the numbers of children classified as obese (BMI between 97th and 99.5th percentiles) were at T1 $n=48$ $(25 / 23)$, at T2 $n=47(25 / 22)$, and at T3 $n=52(27 / 25)$. Additionally, a standardized BMI score (BMI-SDS) [27] was calculated to facilitate comparability across groups.

2.2.2. Socioeconomic Status (SES). In a questionnaire, parents were asked about their formal education and their current occupation. Following a simplified classification scheme based on a system by Blossfeld [29], each parent obtained a score ranging from 0 (= unemployed) to 5 (= highest qualified occupation). The highest score from both parents was used as a criterion for the sample matching.

2.2.3. Processing Speed. Digit-Symbol-Coding from HAWIK4, a German version of the Wechsler Intelligence Scale for Children [30], was used to measure processing speed at T1. According to a code, children had to place the corresponding symbol to either simple geometric shapes (ages 6-7 years) or digits (ages $\geq 8$ years) as quickly as possible. Based on the number of correct items completed in 120 seconds, standardized $T$-scores were calculated. Due to problems during the data collection (e.g., disruption during the test), data from $n=3$ children are missing.

2.2.4. Decision-Making. We used a simplified version of the Hungry Donkey Task (HDT) [5], an age-appropriate version of the IGT [10]. In a computer-game setting, children were asked to assist a hungry donkey to collect as many apples as possible. Opening doors displayed on the computer screen by pressing corresponding keys resulted in certain amounts of "gained" apples (displayed in green) and "lost" apples (displayed in red) at each of 60 trials. To enhance the motivational relevance, children were told that they would win a marble (T1) or a button (T2) if they had collected at least 20 apples at the end of the task. To ensure fairness, all children received this small gift in the end irrespective of the amount of apples they had collected. 

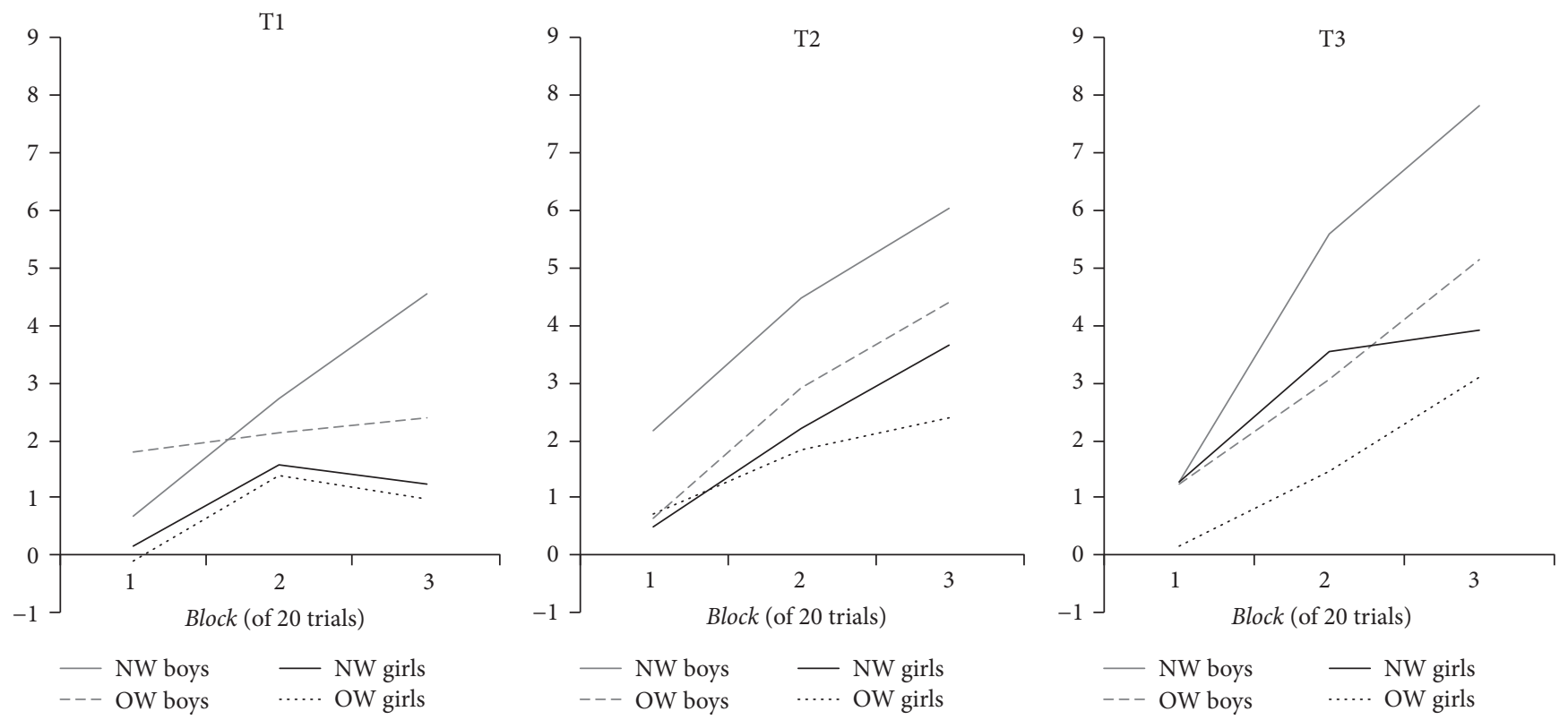

FIGURE 1: Blockwise Hungry Donkey Task (HDT) net scores for normal-weight (NW) and overweight (OW) girls and boys at each wave. Interval between $\mathrm{T} 1$ and $\mathrm{T} 2$ was about 1 year and that between $\mathrm{T} 2$ and $\mathrm{T} 3$ was about 2 years. Blockwise HDT net scores can range from -20 to 20 .

The task was a four-choice version with just two underlying win/loss rules (version AACC by Crone et al. [4]): probabilities for gaining and losing apples were identical for doors $\mathrm{A}$ and $\mathrm{B}$ and for doors $\mathrm{C}$ and $\mathrm{D}$, respectively. This makes the task less complex than the standard fourchoice HDT by Crone and Van Der Molen [5] but more complex than a two-choice gambling task as often used with younger children [e.g., [31-33]]. Also, to ensure an ageappropriate task difficulty, there was a high frequency of losses for all doors (50\% of all trials), based on the finding that children learn to choose advantageous doors faster when they experience frequent losses, compared to less frequent but higher losses [4]. Doors A and B were disadvantageous because choosing them yielded not only higher immediate gains (4 apples per trial) but also higher losses (8-12 apples per $50 \%$ of the trials) resulting in a net loss in the long term; and doors $\mathrm{C}$ and $\mathrm{D}$ were advantageous because choosing them yielded not only lower immediate gains (2 apples per trial) but also lower losses (1-3 apples per $50 \%$ of the trials) resulting in a net gain in the long term.

To examine within-task learning, we calculated a net score difference between advantageous and disadvantageous choices (i.e., choices of doors $\mathrm{C}+\mathrm{D}$ minus doors $\mathrm{A}+\mathrm{B}$ ) for three blocks of 20 trials each. A positive net score indicates a preference for advantageous doors, whereas a negative net score indicates a preference for disadvantageous doors. Moreover, a higher/lower value of the net score indicates relatively fewer/more risky decisions.

2.3. Procedure. Measures were administered as part of a larger longitudinal study on developmental risk factors. Children were tested individually at three waves (T1, T2, and T3) in the years 2012, 2013, and 2015. The interval between
$\mathrm{T} 1$ and T2 was about 1 year and that between T2 and T3 was about 2 years. The data from $\mathrm{T} 1$ and $\mathrm{T} 2$ have been analyzed previously in a prospective study on executive functioning and weight status [15], but these analyses were performed on a larger, not matched sample, using only overall HDT net scores, and there were some issues (essential differences in group sizes and group differences in SES) that we tried to overcome in the present study by using a matched subsample.

Each child was tested individually by a trained experimenter during the morning hours in a quiet room either at school or at home. Parents answered the demographic questions either online or in printed format. At all waves, children received a voucher (worth 7.50 Euros) as reward upon completion of all measures. All procedures were approved by the Research Ethics Board at the University of Potsdam and by the Ministry of Education, Youth, and Sport of the Federal State of Brandenburg.

\section{Results}

HDT net scores (choices of doors $\mathrm{C}+\mathrm{D}$ minus doors $\mathrm{A}+$ B) were analyzed using a mixed-design ANOVA with the between-subject factors weight group (overweight, normalweight) and gender (girls, boys) as well as the within-subject factors block (1-3) and wave (T1, T2, and T3). All analyses were performed using SPSS 24. Where the assumption of sphericity was violated Greenhouse-Geisser corrections were applied. The data are displayed in Figure 1.

Significant main effects were found for all of the four factors: weight group $(F(1,184)=6.87, p<.01$, and partial $\left.\eta^{2}=.04\right)$, gender $\left(F(1,184)=18.33, p<.001\right.$, and partial $\eta^{2}=$ $.09)$, block $\left(F(1.89,348.11)=77.93, p<.001\right.$, and partial $\eta^{2}=$ $.30)$, and wave $\left(F(1.87,343.98)=6.24, p<.01\right.$, and $\operatorname{partial} \eta^{2}=$ 
TABLE 2: Means (and SDs) of blockwise HDT net scores for weight group and gender.

\begin{tabular}{cccccc}
\hline & & NW & OW & Girls & Boys \\
\hline \multirow{3}{*}{ T1 } & Block 1 & $0.45(3.42)$ & $0.89(3.81)^{*}$ & $0.04(3.04)^{*}$ & $1.24(4.01)$ \\
& Block 2 & $2.17(4.86)^{*}$ & $1.77(5.60)^{*}$ & $1.47(4.52)^{*}$ & $2.43(5.80)^{*}$ \\
\hline \multirow{2}{*}{ T2 } & Block 3 & $2.96(6.19)^{*}$ & $1.72(5.71)^{*}$ & $1.11(4.67)^{*}$ & $3.47(6.78)^{*}$ \\
& Block 1 & $1.36(4.49)^{*}$ & $0.68(3.60)$ & $0.6(3.48)^{*}$ & $1.41(4.53)$ \\
& Block 2 & $3.40(6.55)^{*}$ & $2.38(4.62)^{*}$ & $3.02(4.49)^{*}$ & $3.69(6.50)^{*}$ \\
T3 & Block 3 & $4.89(6.78)^{*}$ & $3.45(4.71)^{*}$ & $0.73(3.80)^{*}$ & $5.22(6.93)^{*}$ \\
& Block 1 & $1.28(4.64)^{*}$ & $0.72(3.53)^{*}$ & $2.51(5.00)^{*}$ & $1.24(4.40)$ \\
& Block 2 & $4.62(6.22)^{*}$ & $2.30(5.06)^{*}$ & $3.51(5.81)^{*}$ & $6.43(6.30)^{*}$ \\
\hline
\end{tabular}

Note. Blockwise net scores averaged across weight group or gender, respectively. Net scores can range from -20 to 20. HDT: Hungry Donkey Task; NW: normalweight group; OW: overweight group; T1, T2, and T3: data waves. * Significantly above zero $(p<.05)$.

.03). Averaged over all other factors, normal-weight children had higher net scores than overweight children, and boys had higher net scores than girls. Regarding the significant main effect of block, paired $t$-tests averaged over all other factors revealed a significant increase of the net scores between the first and the second blocks $(t(187)=8.08, p<.001$, and $d=0.62)$, between the first and the third blocks $(t(187)=$ $10.84, p<.001$, and $d=0.86$ ), and between the second and the third blocks $(t(187)=4.97, p<.001$, and $d=0.37)$ even when a Bonferroni-adjusted alpha-level of $p<.016$ (for three comparisons) was applied. Similarly, for the significant main effect of wave, paired $t$-tests averaged over all other factors revealed a significant increase of the net scores between $\mathrm{T} 1$ and T2 $(t(187)=2.71, p<.01$, and $d=0.20)$ and between T1 and T3 $(t(187)=3.50, p<.01$, and $d=0.26)$ but not between $\mathrm{T} 2$ and T3 $(t(187)=1.00, p=.32$, and $d=0.07)$.

The main effects were qualified by significant two-way interactions: blockwise learning within the task was moderated by weight group, gender, and wave. Table 2 depicts blockwise net scores for the three factors. For the significant weight group $\mathrm{x}$ block interaction $(F(1.89,348.11)=3.56, p<$ .05 , and partial $\eta^{2}=.02$ ), post hoc $t$-tests with net scores averaged across gender and waves revealed that the weightgroup difference was not significant in the first block ( $t(186)$ $=0.78, p=.43$, and $d=0.11$ ) but was significant in the second block $(t(186)=2.51, p=.013$, and $d=0.37)$ and in the third block $(t(186)=2.57, p=.013$, and $d=0.38)$ (Bonferroni-adjusted alpha-level: $p<.016$ ), with overweight children producing lower net scores than normal-weight children. Thus, at the beginning of the task, overweight and normal-weight children started similarly, but from the second block onwards, normal-weight children made relatively more advantageous choices than overweight children.

Regarding the significant gender $\mathrm{x}$ block interaction $\left(F(1.89,348.11)=6.33, p<.01\right.$, and partial $\left.\eta^{2}=.03\right)$, post hoc $t$-tests with net scores averaged across weight group and wave revealed that the gender difference was significant in the first block $(t(186)=2.55, p=.012$, and $d=0.37)$, in the second block $(t(186)=3.00, p=.003$, and $d=0.44)$, and in the third block $(t(186)=4.49, p<.001$, and $d=0.66)$, with boys showing higher net scores than girls. This shows that, already early in the task, boys made more advantageous choices than girls, and this difference became quite large from the second block onwards.

For the significant wave $\mathrm{x}$ block interaction $(F(3.62$, $666.12)=4.87, p=.001$, and partial $\eta^{2}=.03$ ), we calculated follow-up repeated-measures ANOVAs for each of the three waves with block (1-3) as the only factor. This revealed a significant main effect of block at all three waves: T1: $F(2,374)$ $=10.34, p<.001$, and partial $\eta^{2}=.05 ; \mathrm{T} 2: F(1.88,350.91)=$ 36.14, $p<.001$, and partial $\eta^{2}=.16$; and T3: $F(1.71,319.22)=$ 45.58, $p<.001$, and partial $\eta^{2}=.20$. Paired-samples $t$-tests showed that net scores of all three blocks differed significantly from each other at each of the three waves (all p's $<.001$ ) with only one exception: at T1, there was no significant difference between the second and the third blocks $(p=.34)$. This displays that, in all three waves, children learned to shift to more advantageous choices within the task. However, whereas at $\mathrm{T} 2$ and $\mathrm{T} 3$ this progress continued till the end of the task, children at T1 significantly improved their performance only from the first to the second block but not from the second to the third block.

All other interactions of the factors were not significant (all p's > .23). In particular, there were no significant wave $\mathrm{x}$ weight group or wave $\mathrm{x}$ gender interactions, indicating that the differences between normal-weight and overweight children or between girls and boys and also the group differences in learning across trials did not differ significantly between waves. Moreover, this shows that groups did not differ significantly in their decision-making development over the 3-year period.

In a second step, we calculated an ANCOVA on the HDT net scores with the same four factors (weight group, gender, block, and wave), including processing speed at $\mathrm{Tl}$ as a covariate. In this ANCOVA, the main effects of weight group and gender as well as the interactions of these factors with block remained significant (all $p$ 's $<.05$ ). However, interestingly, the main effects of block and wave as well as the wave $\mathrm{x}$ block interaction were not significant (all $p$ 's $>.14$ ). Processing speed had a significant main effect $(F(1,180)=4.13, p=$ .04 , and partial $\eta^{2}=.02$ ), but there were no significant interactions of processing speed and any of the other factors (all p's > .30). 
Finally, we did an additional analysis in order to examine longitudinal rank-order stability of decision-making performance in overweight and normal-weight children. For this, we calculated a multigroup path model of the overall HDT net scores (sum of all three blocks). Rank-order stability was very low in both groups, with just one significant path from $\mathrm{T} 1$ to $\mathrm{T} 2$ in the overweight group $(\beta=.27 ; p<.05)$; other paths were not significant (all $p$ 's $>.14$ ).

\section{Discussion}

The aim of this study was to test whether weight-group differences in decision-making under uncertainty can be found already in middle childhood, to examine the age at which they develop, and to explore whether such differences are moderated by children's gender, using a matched sample (94 overweight and 94 normal-weight children matched for age, gender, and SES). Decision-making was measured using the HDT, which is an age-appropriate version of the IGT, and within-task learning was examined via a blockwise analysis (3 blocks of 20 trials) of the net scores that indicated the relative numbers of advantageous and disadvantageous choices. A longitudinal design with three waves (age at T1: 6-9 years) was applied to explore the development of weight-group differences across a 3-year period in middle childhood.

Significant main effects revealed that, averaged over all other factors, net scores were higher (indicating relatively more advantageous choices) for normal-weight than overweight children, for boys than girls, and at T2 than at T1. No significant increase in overall net scores was found from T2 to T3. Blockwise net scores increased significantly from block 1 to block 2 and from block 2 to block 3, indicating learning within the task. Significant interactions revealed that this blockwise learning within the task was moderated by weight group, gender, and wave. Already in the first block, boys had significantly higher net scores than girls, and this gender difference was also present in the other two blocks. Weight-group differences were significant from the second block onwards, with normal-weight children having higher net scores than overweight children. Blockwise learning was evident in all three waves, but whereas in $\mathrm{T} 1$ and $\mathrm{T} 2$ this progress continued till the end of the task, children did not significantly improve their performance from the second to the third block at T1.

Group differences in both the overall net scores and in blockwise learning remained significant after controlling for processing speed, indicating that they did not display mere differences in this basic cognitive ability. However, the main effects of block and wave as well as the wave $\mathrm{x}$ block interaction were not significant anymore after controlling for processing speed, suggesting that more basal cognitive processing influences both the within-task progress and the progress over the 3-year period in decision-making.

Applying these findings to our first hypothesis, we can conclude that, as expected, overweight children were less able than normal-weight children to refrain from high but risky immediate rewards. This proves that the weight-status-related differences in decision-making which have been shown for adults [13] and (pre)adolescents [11, 14] can be found already in 6- to 9-year-old children. The $\mathrm{T} 1$ and $\mathrm{T} 2$ data of the current study come from a subsample of the data analyzed by Groppe and Elsner [15] who examined weight-group differences in several aspects of executive functioning. In the larger sample of the previous study, overall net scores at $\mathrm{T} 1$ were significantly lower in overweight as compared to normal-weight children, but this weight-group difference was not significant at T2. These differences in findings could be due to our use of a matched subsample, because the statistical power of the ANOVA is sensitive to differences in sample size, which were present in the previous study [15], with the normal-weight group being about five times larger than the overweight group. Also, weight-group differences in SES, which were controlled for in the present study, could have flawed the prior results.

The present findings also confirmed our second hypothesis that the weight-group differences would occur only in later trial blocks of the HDT. This matches prior studies in overweight (pre)adolescents (11 to 16 years old) which found lower net scores after 60 trials in the IGT [11] and after 40 trials in the HDT, respectively [14]. Overweight as compared to normal-weight individuals seem to be less able to integrate the feedback about past choices in order to make long-term advantageous choices [13], and our data indicate that this is the case already in middle childhood.

Our third hypothesis that the weight-group differences in decision-making would become larger during a 3-year period within middle childhood was not supported by our data. Neither the differences between overweight and normalweight children nor the gender and weight-group differences in blockwise performance differed significantly between the waves. Thus, we cannot conclude that group differences develop during the studied age period or that gender or overweight has longitudinal effects on decision-making development over the 3-year period. We had theorized that entry to elementary school entails heightened demands for selfregulation in food-related decisions. In this vein, deficits in decision-making would theoretically lead to more unhealthy food intake and weight gain. Although other study designs (e.g., a cross-lagged analysis examining longitudinal relations of decision-making and weight status) would provide a more appropriate analysis to test this prediction, the current study does not provide support for this line of reasoning.

Regarding the development of decision-making, there was some evidence for developmental progress over the 3year period in that overall net scores increased significantly from $\mathrm{T} 1$ to $\mathrm{T} 2$. On average, children did not improve their decision-making performance from T2 to T3, suggesting leveling-off in the development. However, wave differences were not significant anymore after controlling for processing speed, so interpretation regarding the development of decision-making should be cautious. Other study designs would provide a better way to examine development of decision-making abilities in middle childhood, for example, using a larger sample and growth-curve modeling.

Our fourth hypothesis was of exploratory nature, based on inconsistent findings regarding gender differences in decision-making of overweight versus normal-weight adults $[8,22]$. Our data did not support a moderating effect of 
the children's gender on weight-group differences. Both overweight girls and boys showed larger preference for disadvantageous choices than their normal-weight controls. From this, we can conclude that interactive effects of gender and weight group, as found in adults $[8,22]$, might develop later in adolescence.

However, the present data revealed a main effect of gender in that boys had higher net scores than girls. This is in line with prior findings of gender differences in the IGT and its variants (see review [20]). In adults, men typically outperform women in these tasks, although this male advantage depends on the variant used. Gender-specific strategy usage [20] as well as differences in brain mechanisms involved [34] could underlie these gender differences. However, in children and adolescents, findings are less consistent. For instance, higher net scores were found in girls than in boys (3-6 years old), using simplified gambling tasks $[35,36]$, as well as in female than in male adolescents (11-15 years), using the original IGT [37]. In contrast, in a study using the HDT, boys outperformed girls (7-15 years old) [4], and at an even younger age, there was a statistical trend for a male advantage in a simplified IGT only in 3-year-olds, but there was no evidence for gender differences in 4-year-olds [31,38]. The present study found a male advantage in decision-making of 6- to 12-year-olds over a 3-year period using a simplified HDT, but further research is needed to investigate the mechanisms (e.g., participants' focus on different strategies) [20] that underlie the frequently found gender differences in decision-making tasks and their development in childhood and adolescence.

In the present study, effects of processing speed could be ruled out for gender- and weight-group-related differences in decision-making. We did not find differences in processing speed between overweight and normal-weight children or between boys and girls, respectively, and processing speed was not involved in any of the interactions of the factors, indicating that the differences in within-task learning of decision-making performance were not based on differences in processing speed in this sample.

An additionally calculated path model of overall HDT net scores revealed very low rank-order stability of decisionmaking performance in both weight groups. This means that, within the weight groups, children changed their rank position in decision-making performance relatively to the other children. However, the significant group differences in the ANOVA indicate that between-group differences were larger than within-group variance, and the nonsignificant interaction of group and wave suggests that group differences were stable over the study period. Even so, the somewhat surprising finding indicates a large interindividual variance in the intraindividual development of decision-making, an issue that should be examined and addressed in future studies.

To our knowledge, the present study is the first to investigate effects of gender and weight group on the development of decision-making in middle childhood and examining the blockwise progress within the task. Strengths of our study include the longitudinal design, the large sample size, and the carefully matched control group, which allowed for weightgroup comparisons under control of gender, age, and SES.
However, a limitation is that we used just one simplified version of the HDT, missing the chance to explore which aspects of decision-making actually make the difference. Verbeken et al. [14] found the largest weight-group differences not in the regular version of the HDT but in a reversed version, in which losses occurred consistently and gains occurred more or less frequently. Thus, overweight children's decision-making deficits may result from insensitivity to future consequences rather than hypersensitivity to rewards. In the simplified version of the HDT which was used in the current study, there were just two kinds of underlying rules, and decks differed only in the magnitude of losses, while frequency of losses was the same for all decks. Thus, interpretation regarding specific decision-making processes is limited. Another limitation is the relatively small amount of trials we used. Even though in our study weight-group differences in blockwise learning emerged at the second block (after 20 trials), weight-group differences in prior studies primarily occurred from 60 trials onwards (e.g., [11]). Thus, by adding more trials, even larger group differences might have had been detected.

In conclusion, the present study demonstrated that differences in within-task learning of decision-making between normal-weight and overweight individuals are already present in 6- to 12-year-olds. Impaired self-regulated decision-making, based on insensitivity to negative future consequences, might be a risk factor for the development of overweight. Yet, causality cannot be inferred from the recent study, and future research is needed to understand the causal directions of the associations, the underlying mechanisms, and the developmental changes from childhood to adulthood. Moreover, it should be kept in mind that that there are manifold factors contributing to the etiology of overweight and obesity and that these factors interact in a complex matter that might differ between individuals at different ages. However, given the high prevalence of overweight in children and its strong predictive effect for overweight and other healthrelated problems in adulthood [39], the present findings are an important contribution to the mission to explore the developmental impact of decision-making and other selfregulatory processes on weight status in our obesogenic environment.

\section{Conflicts of Interest}

The authors declare that there are no conflicts of interest regarding the publication of this article.

\section{Acknowledgments}

The authors thank the schools, the children and their families, and all team members for their contribution to the study. This work was supported by the German Research Foundation (DFG) under Grant no. 1668/1.

\section{References}

[1] M. Ng, T. Fleming, M. Robinson, and et al, "Global, regional, and national prevalence of overweight and obesity in children and adults during 1980-2013: a systematic analysis for the 
Global Burden of Disease Study 2013," The Lancet, vol. 384, no. 9945, pp. 766-781, 2014.

[2] C. Davis, R. D. Levitan, P. Muglia, C. Bewell, and J. L. Kennedy, "Decision-making deficits and overeating: A risk model for obesity," Obesity Research, vol. 12, no. 6, pp. 929-935, 2004.

[3] W. Stroebe, G. M. Van Koningsbruggen, E. K. Papies, and H. Aarts, "Why most dieters fail but some succeed: A goal conflict model of eating behavior," Psychological Review, vol. 120, no. 1, pp. 110-138, 2013.

[4] E. A. Crone, S. A. Bunge, H. Latenstein, and M. W. Van Der Molen, "Characterization of children's decision making: Sensitivity to punishment frequency, not task complexity," Child Neuropsychology, vol. 11, no. 3, pp. 245-263, 2005.

[5] E. A. Crone and M. W. Van Der Molen, "Developmental changes in real life decision making: Performance on a gambling task previously shown to depend on the ventromedial prefrontal cortex," Developmental Neuropsychology, vol. 25, no. 3, pp. 251279, 2004.

[6] A. Bechara, H. Damasio, and A. R. Damasio, "Emotion, decision making and the orbitofrontal cortex," Cerebral Cortex, vol. 10, no. 3, pp. 295-307, 2000.

[7] K. R. S. Reinert, E. K. Po'e, and S. L. Barkin, "The relationship between executive function and obesity in children and adolescents: a systematic literature review," Journal of Obesity, vol. 2013, Article ID 820956, 10 pages, 2013.

[8] A. Horstmann, F. P. Busse, D. Mathar et al., "Obesity-related differences between women and men in brain structure and goal-directed behavior," Frontiers in Human Neuroscience, no. JUNE, article no. 58, 2011.

[9] J. I. Cohen, K. F. Yates, M. Duong, and A. Convit, "Obesity, orbitofrontal structure and function are associated with food choice: A cross-sectional study," BMJ Open, vol. 1, no. 2, Article ID e000175, 2011.

[10] A. Bechara, A. R. Damasio, H. Damasio, and S. W. Anderson, "Insensitivity to future consequences following damage to human prefrontal cortex," Cognition, vol. 50, no. 1-3, pp. 7-15, 1994.

[11] A. Verdejo-García, M. Pérez-Expósito, and J. Schmidt-RíoValle, "Selective alterations within executive functions in adolescents with excess weight," Obesity, vol. 18, no. 8, pp. 1572-1578, 2010.

[12] S. Fitzpatrick, S. Gilbert, and L. Serpell, "Systematic review: Are overweight and obese individuals impaired on behavioural tasks of executive functioning?" Neuropsychology Review, vol. 23, no. 2, pp. 138-156, 2013.

[13] M. Wu, T. Brockmeyer, M. Hartmann, M. Skunde, W. Herzog, and H.-C. Friederich, "Reward-related decision making in eating and weight disorders: A systematic review and metaanalysis of the evidence from neuropsychological studies," Neuroscience \& Biobehavioral Reviews, vol. 61, pp. 177-196, 2016.

[14] S. Verbeken, C. Braet, G. Bosmans, and L. Goossens, "Comparing decision making in average and overweight children and adolescents," International Journal of Obesity, vol. 38, no. 4, pp. 547-551, 2014.

[15] K. Groppe and B. Elsner, "Executive function and weight status in children: A one-year longitudinal perspective," Child Neuropsychology, vol. 23, no. 2, pp. 129-147, 2017.

[16] L. McLaren, "Socioeconomic status and obesity," Epidemiologic Reviews, vol. 29, no. 1, pp. 29-48, 2007.

[17] F. Mata, I. Sallum, D. M. Miranda, A. Bechara, and L. F. MalloyDiniz, "Do general intellectual functioning and socioeconomic status account for performance on the Children's Gambling Task?" Frontiers in Neuroscience, no. 7, Article ID Article 68, 2013.

[18] C. Davis, K. Patte, C. Curtis, and C. Reid, "Immediate pleasures and future consequences. A neuropsychological study of binge eating and obesity," Appetite, vol. 54, no. 1, pp. 208-213, 2010.

[19] A. Brogan, D. Hevey, G. O'Callaghan, R. Yoder, and D. O'Shea, "Impaired decision making among morbidly obese adults," Journal of Psychosomatic Research, vol. 70, no. 2, pp. 189-196, 2011.

[20] R. van den Bos, J. Homberg, and L. de Visser, "A critical review of sex differences in decision-making tasks: Focus on the Iowa Gambling Task," Behavioural Brain Research, vol. 238, no. 1, pp. 95-108, 2013.

[21] R. von Kries, A. Beyerlein, M. J. Müller et al., "Different age-specific incidence and remission rates in pre-school and primary school suggest need for targeted obesity prevention in childhood," International Journal of Obesity, vol. 36, no. 4, pp. 505-510, 2012.

[22] G. Koritzky, E. Yechiam, I. Bukay, and U. Milman, "Obesity and risk taking. A male phenomenon," Appetite, vol. 59, no. 2, pp. 289-297, 2012.

[23] R. E. Weller, E. W. Cook III, K. B. Avsar, and J. E. Cox, “Obese women show greater delay discounting than healthy-weight women," Appetite, vol. 51, no. 3, pp. 563-569, 2008.

[24] I. Paus-Hasebrink and J. Kulterer, "Commercialization of childhood," in Handbuch Kinder und Medie, A. Tillmann, K. Hugger, and T. Hug, Eds., pp. 47-57, 2014.

[25] E. A. Crone and M. W. Van Der Molen, "Development of decision making in school-aged children and adolescents: Evidence from heart rate and skin conductance analysis," Child Development, vol. 78, no. 4, pp. 1288-1301, 2007.

[26] P. D. Zelazo and S. M. Carlson, "Hot and Cool Executive Function in Childhood and Adolescence: Development and Plasticity," Child Development Perspectives, vol. 6, no. 4, pp. 354360, 2012.

[27] K. Kromeyer-Hauschild, M. Wabitsch, D. Kunze, F. Geller et al., "Body mass index percentiles for childhood and adolescence based on several German samples," Monatsschrift Kinderheilkd, vol. 149, pp. 807-818, 2001.

[28] B. M. Kurth and A. Schaffrath Rosario, "The prevalence of overweight and obese children and adolescents living in Germany," Bundesgesundheitsblatt Gesundheitsforschung Gesundheitsschutz, vol. 50, no. 5-6, pp. 736-743, 2007.

[29] B. Schimpl-Neimanns, Microdata tools: Blossfeld's Occupational Classification for the 1973-1998 Microcensus, 2003.

[30] F. Petermann and U. Petermann, Hamburg-Wechsler-Intelligenztest für Kinder - IV, Bern: Huber, 2008, Wechsler intelligence scale for children - German version.

[31] A. Kerr and P. D. Zelazo, "Development of "hot" executive function: The children's gambling task," Brain and Cognition, vol. 55, no. 1, pp. 148-157, 2004.

[32] G. Andrews and J. Moussaumai, "Improving children's affective decision making in the Children's Gambling Task," Journal of Experimental Child Psychology, vol. 139, pp. 18-34, 2015.

[33] K. M. Bunch, G. Andrews, and G. S. Halford, "Complexity effects on the children's gambling task," Cognitive Development, vol. 22, no. 3, pp. 376-383, 2007.

[34] K. I. Bolla, D. A. Eldreth, J. A. Matochik, and J. L. Cadet, "Sex-related differences in a gambling task and its neurological correlates," Cerebral Cortex, vol. 14, no. 11, pp. 1226-1232, 2004. 
[35] R. M. Heilman, A. C. Miu, and O. Benga, "Developmental and sex-related differences in preschoolers' affective decision making," Child Neuropsychology, vol. 15, no. 1, pp. 73-84, 2009.

[36] N. Garon and C. Moore, "Complex decision-making in early childhood," Brain and Cognition, vol. 55, no. 1, pp. 158-170, 2004.

[37] M. d'Acremont and M. Van der Linden, "Gender differences in two decision-making tasks in a community sample of adolescents," International Journal of Behavioral Development, vol. 30, no. 4, pp. 352-358, 2006.

[38] N. Garon and C. Moore, "Developmental and gender differences in future-oriented decision-making during the preschool period," Child Neuropsychology, vol. 13, no. 1, pp. 46-63, 2007.

[39] J. Steinberger, A. Moran, C.-P. Hong, D. R. Jacobs Jr., and A. R. Sinaiko, "Adiposity in childhood predicts obesity and insulin resistance in young adulthood," Journal of Pediatrics, vol. 138, no. 4, pp. 469-473, 2001. 


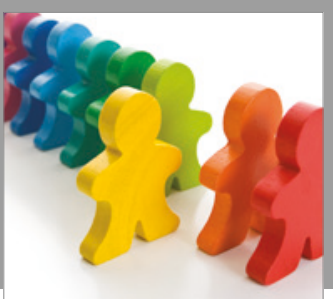

Autism

Research and Treatment
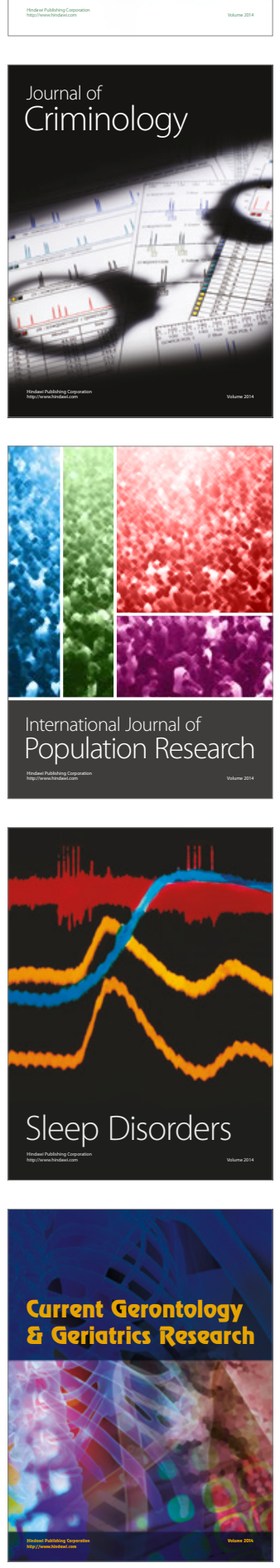

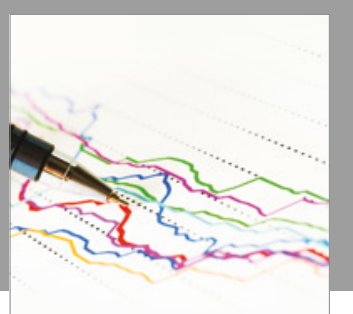

Economics

Research International

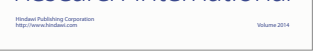

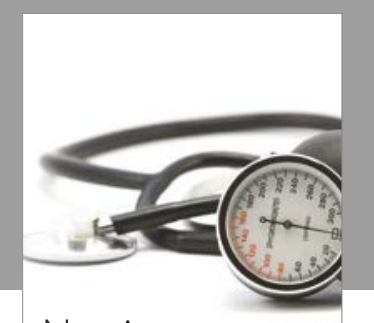

Nursing

Research and Practice

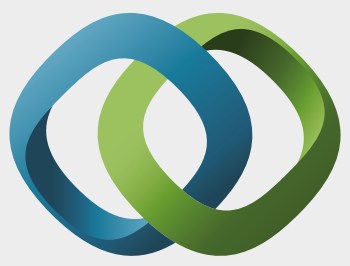

\section{Hindawi}

Submit your manuscripts at

https://www.hindawi.com
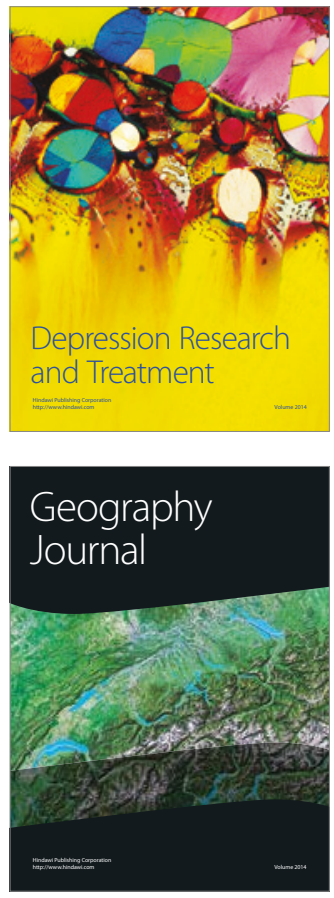
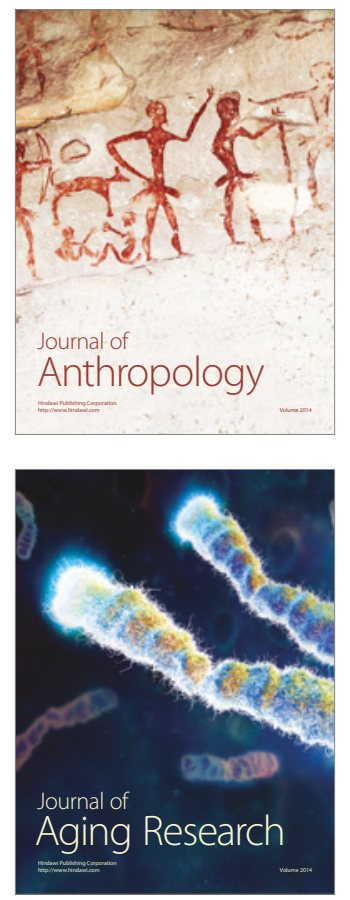
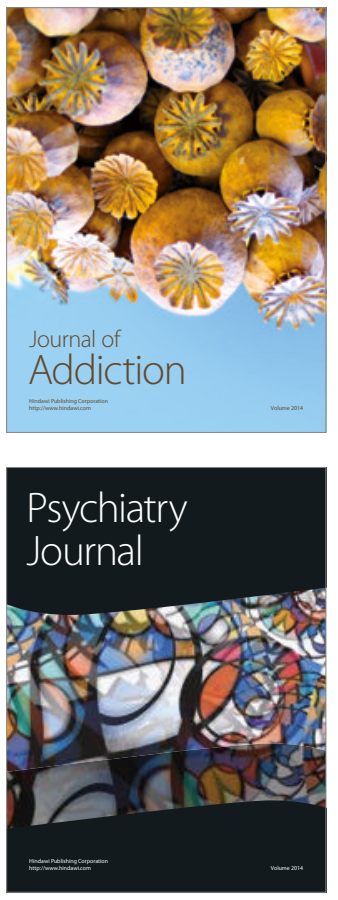

Child Development

Research

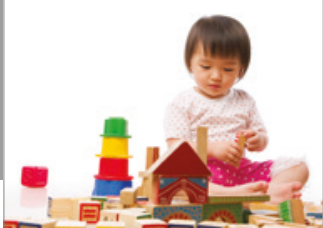

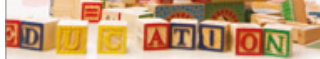
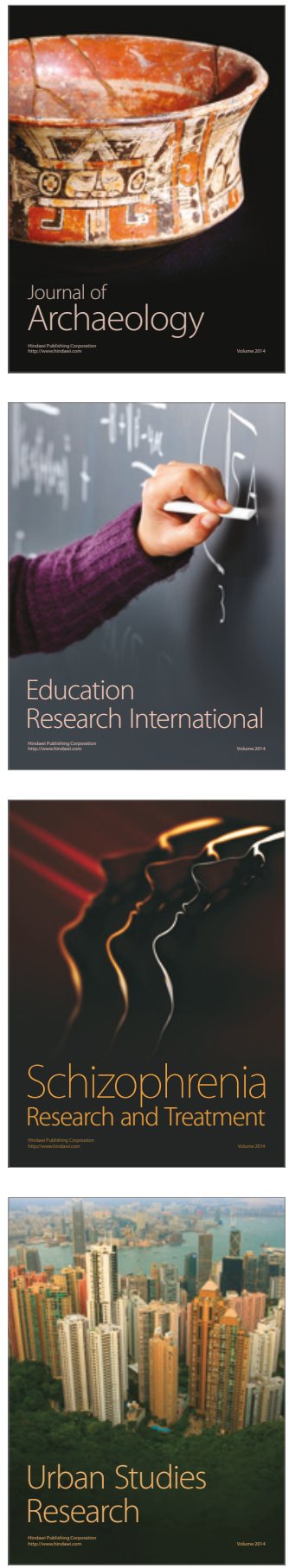